\title{
M-polynomial and related degree-based topological indices of the third type of hex-derived network
}

\author{
Shibsankar Das, Shikha Rai \\ Department of Mathematics, Institute of Science, Banaras Hindu University, Varanasi-221005, Uttar Pradesh, India \\ shib.iitm@gmail.com, shikharai48@gmail.com
}

PACS 02.10.0x, 61.48.-c

DOI 10.17586/2220-8054-2020-11-3-267-274

\begin{abstract}
In the field of chemical graph theory, a topological index is a real number which is correlated with the various physical properties, biological activities and chemical reactivities of molecular graphs. In recent trends, M-polynomials are used to compute numerous degree-based topological indices. Hex-derived networks have a wide range of applications in pharmaceutical sciences, electronics and communication networks. In this paper, we would like to determine a general form of M-polynomial for the third type of hex-derived network of dimension $n$ and hence generate the related degree-based topological indices. Additionally, we plot the M-polynomial and all the related degree-based topological indices for several $n$.
\end{abstract}

Keywords: Third type of hex-derived network, Degree-based topological indices, M-polynomial, Graph polynomial.

Received: 13 April 2020

Revised: 26 April 2020

\section{Introduction}

Let $G=(V, E)$ be a simple connected graph, where $V=V(G)$ and $E=E(G)$ respectively denote the vertex set and the edge set which contains unordered pairs of vertices. The degree of a vertex $u \in V(G)$ in $G$ is the number of vertices that are adjacent to $u$ and is denoted by $d(u)[1]$.

\subsection{A brief review on topological indices and M-polynomials}

Mathematical aspects of chemical compounds and their behaviors are being investigated in the area of Chemical Graph Theory (CGT). For a given chemical compound, each atom is represented by a vertex and each chemical bond between the atoms is represented by an edge to form a graph. Several chemical applications of graphs and the connection between graph theory and chemistry have been reported in [2-5]. In the graph-theoretical representation of a molecule, we can study various biological activities, physical properties, chemical reactivities and other properties of chemical compounds. In the area of CGT, a topological index (also known as a graph-theoretic index or connectivity index) specifies the properties of a molecular structure. Generally, it is a numerical representation of a molecule, which is used in the development of Quantitative Structure Activity Relationships (QSARs) and Quantitative Structure Property Relationships (QSPRs). For more details please refer to [6,7].

The topological indices are usually partitioned into degree-based topological indices [8], distance-based topological indices [9], degree and distance-based topological indices [10] and counting related topological indices [11]. These indices describe the physical, biological, chemical and other properties of various structures. Generally, by the definitions of the respective topological indices, we can calculate their numeric values. Instead, a general method can be found to directly produce the different topological indices of a particular class. Keeping this idea in mind, the concept of polynomials [12] is introduced in graph theory. By constructing a general polynomial corresponding to a given structure, one can derive several topological indices by differentiating or integrating (or a different combination of both) the corresponding polynomial.

In literature, several such polynomials are proposed. Some of which are the matching polynomial [13], the Clar covering polynomial (also known as the Zhang-Zhang polynomial) [14], the Schultz polynomial [15], the Tutte polynomial [16], the Hosoya polynomial [17], etc. Among all of these polynomials, the Hosoya polynomial is used for finding the distance-based topological indices such as the Wiener index [18].

The degree-based topological indices play a major role to understand various properties of a molecular structure. In the recent studies, the idea of M-polynomial is proposed by Deutsch and Klavžar [19] and determined how to evaluate several degree-based topological indices. Please refer to the papers [20-24], in each of which the M-polynomial corresponding to a chemical network is derived and henceforth their related degree-based topological indices are evaluated. 
Definition 1 ([19]). For a simple connected graph $G$, the expression

$$
M(G ; x, y)=\sum_{\delta \leq i \leq j \leq \Delta} m_{i, j}(G) x^{i} y^{j}
$$

is known as the M-polynomial of a graph $G$, where $\delta=\min \{d(u) \mid u \in V(G)\}, \Delta=\max \{d(u) \mid u \in V(G)\}$ and $m_{i, j}(G)$ is the number of edges $u v \in E(G)$ such that $d(u)=i, d(v)=j(i, j \geq 1)$.

A degree-based topological index of a graph $G$ is one of a kind of graph invariants, which is denoted as $I(G)$ and as discussed in [25], it can be written as:

$$
I(G)=\sum_{i \leq j} m_{i, j}(G) f(i, j)
$$

Theorem 2 ( [19], Theorems 2.1, 2.2). Let $G$ be a simple connected graph.

(1) If $I(G)=\sum_{e=u v \in E(G)} f(d(u), d(v))$, where $f(x, y)$ is a polynomial in $x$ and $y$, then

$$
I(G)=\left.f\left(D_{x}, D_{y}\right)(M(G ; x, y))\right|_{x=y=1} .
$$

(2) If $I(G)=\sum_{e=u v \in E(G)} f(d(u), d(v))$, where $f(x, y)=\sum_{i, j \in \mathbb{Z}} \alpha_{i, j} x^{i} y^{j}$, then $I(G)$ can be obtained from $M(G ; x, y)$ using the operators $D_{x}, D_{y}, S_{x}$, and $S_{y}$.

(3) If $I(G)=\sum_{e=u v \in E(G)} f(d(u), d(v))$, where $f(x, y)=\frac{x^{r} y^{s}}{(x+y+\alpha)^{t}}$, where $r, s \geq 0$, $t \geq 1$ and $\alpha \in \mathbb{Z}$, then

$$
I(G)=\left.S_{x}^{t} Q_{\alpha} J D_{x}^{r} D_{y}^{s}(M(G ; x, y))\right|_{x=1} .
$$

\subsection{Associated degree-based topological indices}

In this section, we discuss a brief about certain degree-based topological indices that can be derived from the M-polynomial of our considered network. Gutman and Trinajstić [26] proposed the Zagreb indices in 1972. These indices help in determining the total $\pi$-electron energy which is related to the thermodynamic stability of a molecule.

\begin{tabular}{|c|c|c|c|}
\hline $\begin{array}{l}\text { Sl. } \\
\text { No. }\end{array}$ & Topological Index & Notation & Formula of Topological Indices \\
\hline 1. & $\begin{array}{l}\text { First Zagreb In- } \\
\operatorname{dex}[26]\end{array}$ & $M_{1}(G)$ & $M_{1}(G)=\sum_{u v \in E(G)}(d(u)+d(v))$ \\
\hline 2. & $\begin{array}{l}\text { Second Zagreb In- } \\
\text { dex [26] }\end{array}$ & $M_{2}(G)$ & $M_{2}(G)=\sum_{u v \in E(G)}(d(u) d(v))$ \\
\hline 3. & $\begin{array}{l}\text { Modified Second Za- } \\
\text { greb Index [27] }\end{array}$ & ${ }^{m} M_{2}(G)$ & ${ }^{m} M_{2}(G)=\sum_{u v \in E(G)} \frac{1}{d(u) d(v)}$ \\
\hline 4. & $\begin{array}{l}\text { General Randić In- } \\
\text { dex [28] }\end{array}$ & $R_{\alpha}(G)$ & $R_{\alpha}(G)=\sum_{u v \in E(G)}(d(u) d(v))^{\alpha}$ \\
\hline 5. & $\begin{array}{l}\text { Inverse Randić In- } \\
\text { dex [29] }\end{array}$ & $R R_{\alpha}(G)$ & $R R_{\alpha}(G)=\sum_{u v \in E(G)} \frac{1}{(d(u) d(v))^{\alpha}}$ \\
\hline 6. & $\begin{array}{l}\text { Symmetric Division } \\
\text { (Deg) Index [30] }\end{array}$ & $S D D(G)$ & $S D D(G)=\sum_{u v \in E(G)}\left\{\frac{\min (d(u), d(v))}{\max (d(u), d(v))}+\frac{\max (d(u), d(v))}{\min (d(u), d(v))}\right\}$ \\
\hline 7. & Harmonic Index [31] & $H(G)$ & $H(G)=\sum_{u v \in E(G)} \frac{2}{d(u)+d(v)}$ \\
\hline 8. & $\begin{array}{l}\text { Inverse Sum (Indeg) } \\
\text { Index [30] }\end{array}$ & $I S I(G)$ & $I S I(G)=\sum_{u v \in E(G)} \frac{d(u) d(v)}{d(u)+d(v)}$ \\
\hline 9. & $\begin{array}{l}\text { Augmented Zagreb } \\
\text { Index [32] }\end{array}$ & $A Z(G)$ & $A Z(G)=\sum_{u v \in E(G)}\left\{\frac{d(u) d(v)}{d(u)+d(v)-2}\right\}^{3}$ \\
\hline
\end{tabular}
The Zagreb indices give higher weightage to the inner edges and vertices rather than the outer edges and vertices. With the opposite intuition, the definitions of modified Zagreb indices are proposed in [27].

TABLE 1. Formula for degree-based topological indices of a graph $G$ 
Another very well-known degree-based topological index is the Randić index. It is introduced by Milan Randić [33] in 1975. It is also known as a connectivity index or branching index. This index has wide applications in the field of drug design. Two decades later, observing the advancement of Randić index, the mathematicians Bollobás and Erdôs [28], and Amić et al. [29] introduced the generalized version of the Randić index (for an arbitrary real number $\alpha$ ) in 1998, which is known as general Randić index. For $\alpha=-\frac{1}{2}, R_{\alpha}$ becomes a Randić index [33]; for $\alpha=1$, $R_{\alpha}$ becomes a second Zagreb index; and for $\alpha=-1, R_{\alpha}$ turns out to be a modified second Zagreb index. Around 2010, the symmetric division (deg) index is proposed in [30] to determine the total surface area of polychlorobiphenyls. Whereas, the inverse sum (indeg) index [30,34] predicts the total surface area of octane isomers. For the study of the heat of formation of alkanes, the augmented Zagreb index [32] is beneficial. Table 1 lists the formulas of the different degree-based topological indices (of a graph $G$ ) which are associated with M-polynomial.

\subsection{The hex-derived network of type 3 of dimension $n$}

The Hexagonal network of dimension $n$ is introduced in [35] and is denoted by $H X[n]$. It has several applications in the image processing to model the benzenoid hydrocarbons in the field of chemistry, wireless sensor networks and computer graphics. Later in 2008, the hex-derived network of type 1 (HDN1) and hex-derived network of type 2 (HDN2) of dimension $n$ (where $n$ denotes the number of vertices in a side of the HDN structure) are constructed from the $H X[n]$ [36]. Again in 2017, based on the structure of the $H X[n]$, Raj and George [37] have derived a new network called hex-derived network of type 3 of dimension $n$ (denoted as $H D N 3[n]$ ). Fig. 1 depicts the pictorial representation of the third type of hex-derived network of dimension 4 (that is, HDN3[4]). The metric dimension of $H D N 3[n]$ has a wide range of applications in robotics, networking etc.

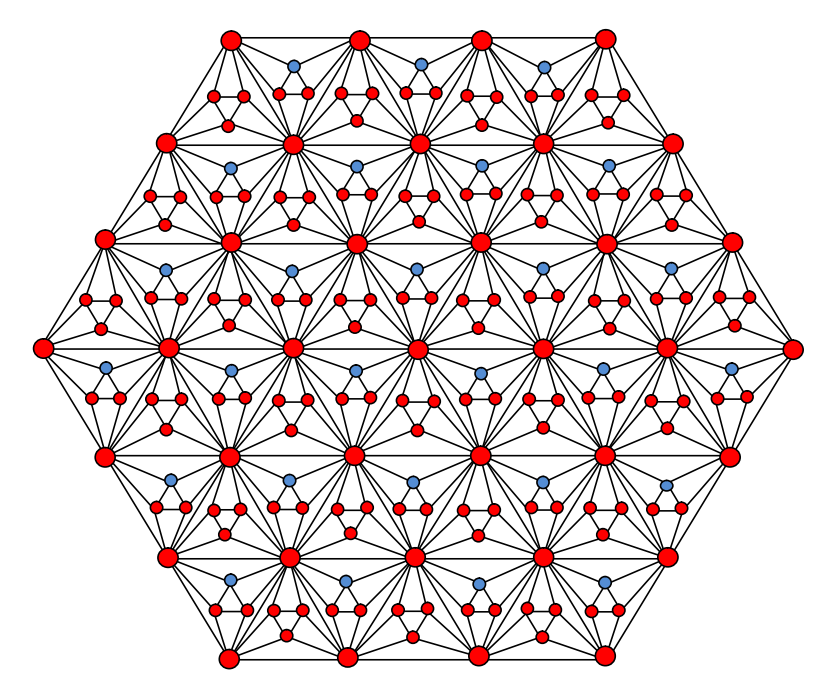

FIG. 1. Type 3 hex-derived network of dimension 4 (HDN3[4])

\subsection{Our contribution and roadmap}

Recently, Wei et. al. [38] computed several degree-based topological indices of HDN3[n]. The indices are calculated separately as well as directly by using their degree-based formulas mentioned in Table 1. As an alternative of the above, we derive a closed-form of M-polynomial for the HDN3[n] in Section 2. Henceforward, we calculate the related degree-based topological indices of the network (for different values of $n$ ) via using the M-polynomial. Moreover, the graphical representation of the M-polynomial and related topological indices for different dimensions of the network are plotted in Section 3.

\section{Deriving a M-polynomial for $\boldsymbol{H D N} 3[n]$ network}

Theorem 3. Let HDN3 $[n]$ be the third type of hex-derived network of dimension $n(\geq 4)$. Then a M-polynomial of HDN3 $[n]$ is

$M(H D N 3[n] ; x, y)=\left(18 n^{2}-36 n+18\right) x^{4} y^{4}+24 x^{4} y^{7}+(36 n-72) x^{4} y^{10}+\left(36 n^{2}-108 n+84\right) x^{4} y^{18}+12 x^{7} y^{10}+$ $6 x^{7} y^{18}+(6 n-18) x^{10} y^{10}+(12 n-24) x^{10} y^{18}+\left(9 n^{2}-33 n+30\right) x^{18} y^{18}$. 
Proof. Let us first enumerate the cardinality of the vertex set and the edge set of the $H D N 3[n]$ by observing the Fig. 1 of $H D N 3[4]$.

$$
\begin{gathered}
|V(H D N 3[n])|=21 n^{2}-39 n+19, \text { and } \\
|E(H D N 3[n])|=63 n^{2}-123 n+60 .
\end{gathered}
$$

Observe that, based on the degree of vertices of $H D N 3[n]$, the network consists of 4, 7, 10 and 18 degrees vertices only, and each of whose cardinality is $18(n-1)^{2}, 6,6(n-2)$ and $3 n^{2}-9 n+7$, respectively. Also, we partition the edge set $E(H D N 3[n])$ of $H D N 3[n]$ into nine disjoint sub-classes depending on the degrees of the end vertices of each edge, as: $E_{\{i, j\}}=\{e=u v \in E(H D N 3[n]): d(u)=i, d(v)=j\}$ where $\{i, j\}=\{4,4\},\{4,7\},\{4,10\}$, $\{4,18\},\{7,10\},\{7,18\},\{10,10\},\{10,18\},\{18,18\}$. And the cardinality of each of the above edge sets is given by $\left|E_{\{4,4\}}\right|=18 n^{2}-36 n+18,\left|E_{\{4,7\}}\right|=24,\left|E_{\{4,10\}}\right|=36 n-72,\left|E_{\{4,18\}}\right|=36 n^{2}-108 n+84,\left|E_{\{7,10\}}\right|=12$, $\left|E_{\{7,18\}}\right|=6,\left|E_{\{10,10\}}\right|=6 n-18,\left|E_{\{10,18\}}\right|=12 n-24,\left|E_{\{18,18\}}\right|=9 n^{2}-33 n+30$. Observe that the hexderive network structure does not have any edge $u v$ such that $d(u)=7=d(v)$ and as a consequence $\left|E_{\{7,7\}}\right|=0$. Therefore, by the basic definition of M-polynomial, the M-polynomial of $H D N 3[n]$ is

$$
\begin{aligned}
& M(H D N 3[n] ; x, y) \\
& =\sum_{i \leq j} m_{i, j} x^{i} y^{j}, \text { where } i, j \in\{4,7,10,18\} \\
& =\left|E_{\{4,4\}}\right| x^{4} y^{4}+\left|E_{\{4,7\}}\right| x^{4} y^{7}+\left|E_{\{4,10\}}\right| x^{4} y^{10}+\left|E_{\{4,18\}}\right| x^{4} y^{18}+\left|E_{\{7,10\}}\right| x^{7} y^{10} \\
& \quad+\left|E_{\{7,18\}}\right| x^{7} y^{18}+\left|E_{\{10,10\}}\right| x^{10} y^{10}+\left|E_{\{10,18\}}\right| x^{10} y^{18}+\left|E_{\{18,18\}}\right| x^{18} y^{18} \\
& =\left(18 n^{2}-36 n+18\right) x^{4} y^{4}+24 x^{4} y^{7}+(36 n-72) x^{4} y^{10}+\left(36 n^{2}-108 n+84\right) x^{4} y^{18} \\
& \quad+12 x^{7} y^{10}+6 x^{7} y^{18}+(6 n-18) x^{10} y^{10}+(12 n-24) x^{10} y^{18}+\left(9 n^{2}-33 n+30\right) x^{18} y^{18} .
\end{aligned}
$$

TABLE 2. Formulas for degree-based topological indices derived from the M-polynomial of a graph $G$

\begin{tabular}{|l|l|c|c|c|}
\hline $\begin{array}{l}\text { Sl. } \\
\text { No. }\end{array}$ & Topological Index & Notation & $\mathbf{f}(\mathbf{x , y})$ & Derivation from $(M(G ; x, y))$ \\
\hline 1. & First Zagreb Index & $M_{1}(G)$ & $x+y$ & $\left.\left(D_{x}+D_{y}\right)(M(G ; x, y))\right|_{x=y=1}$ \\
\hline 2. & Second Zagreb Index & $M_{2}(G)$ & $x y$ & $\left.\left(D_{x} D_{y}\right)(M(G ; x, y))\right|_{x=y=1}$ \\
\hline 3. & $\begin{array}{l}\text { Modified Second Za- } \\
\text { greb Index }\end{array}$ & $m_{2} M_{2}(G)$ & $\frac{1}{x y}$ & $\left.\left(S_{x} S_{y}\right)(M(G ; x, y))\right|_{x=y=1}$ \\
\hline 4. & $\begin{array}{l}\text { General Randić In- } \\
\text { dex }\end{array}$ & $R_{\alpha}(G)$ & $(x y)^{\alpha}$ & $\left.\left(D_{x}^{\alpha} D_{y}^{\alpha}\right)(M(G ; x, y))\right|_{x=y=1}$ \\
\hline 5. & Inverse Randić Index & $R R_{\alpha}(G)$ & $\frac{1}{(x y)^{\alpha}}$ & $\left.\left(S_{x}^{\alpha} S_{y}^{\alpha}\right)(M(G ; x, y))\right|_{x=y=1}$ \\
\hline 6. & $\begin{array}{l}\text { Symmetric Division } \\
\text { (Deg) Index }\end{array}$ & $S D D(G)$ & $\frac{x^{2}+y^{2}}{x y}$ & $\left.\left(D_{x} S_{y}+D_{y} S_{x}\right)(M(G ; x, y))\right|_{x=y=1}$ \\
\hline 7. & Harmonic Index & $H(G)$ & $\frac{2}{x+y}$ & $\left.2 S_{x} J(M(G ; x, y))\right|_{x=1}$ \\
\hline 8. & $\begin{array}{l}\text { Inverse Sum (Indeg) } \\
\text { Index }\end{array}$ & $I S I(G)$ & $\frac{x y}{x+y}$ & $\left.S_{x} J D_{x} D_{y}(M(G ; x, y))\right|_{x=1}$ \\
\hline 9. & $\begin{array}{l}\text { Augmented Zagreb } \\
\text { Index }\end{array}$ & $A Z(G)$ & $\left(\frac{x y}{x+y-2}\right)^{3}$ & $\left.S_{x}^{3} Q_{-2} J D_{x}^{3} D_{y}^{3}(M(G ; x, y))\right|_{x=1}$ \\
\hline
\end{tabular}

To calculate the concerned topological indices (as mentioned in Table 1) of a given graph $G$ from the $M(G ; x, y)$, the formulas of derivations in terms of integral or derivative (or both integral and derivative) are listed in Table 2 [19], where $D_{x}=x \frac{\partial(f(x, y))}{\partial x}, D_{y}=y \frac{\partial(f(x, y))}{\partial y}, S_{x}=\int_{0}^{x} \frac{f(t, y)}{t} d t, S_{y}=\int_{0}^{y} \frac{f(x, t)}{t} d t, J(f(x, y))=f(x, x)$, and $Q_{\alpha}(f(x, y))=x^{\alpha} f(x, y), \alpha \neq 0$.

The following theorem determines the values of the related degree-based topological indices of the $H D N 3[n]$ from the above proposed M-polynomial $M(H D N 3[n] ; x, y)$.

Theorem 4. Let HDN3 $[n]$ be the third type of hex-derived network of dimension $n(\geq 4)$. Then

(1) $M_{1}(H D N 3[n])=6\left(210 n^{2}-482 n+275\right)$. 
(2) $M_{2}(H D N 3[n])=12\left(483 n^{2}-1237 n+777\right)$.

(3) ${ }^{m} M_{2}(H D N 3[n])=\frac{1}{16}\left(18 n^{2}-36 n+18\right)+\frac{24}{28}+\frac{1}{40}(36 n-72)+\frac{1}{72}\left(36 n^{2}-108 n+84\right)+\frac{12}{70}+\frac{6}{126}+$ $\frac{1}{100}(6 n-18)+\frac{1}{180}(12 n-24)+\frac{1}{324}\left(9 n^{2}-33 n+30\right)=\frac{119}{72} n^{2}-\frac{1907}{675} n+\frac{50921}{37800}$.

(4) $R_{\alpha}(H D N 3[n])=4^{2 \alpha}\left(18 n^{2}-36 n+18\right)+24 \times 28^{\alpha}+40^{\alpha}(36 n-72)+72^{\alpha}\left(36 n^{2}-108 n+84\right)+12 \times$ $70^{\alpha}+6 \times 126^{\alpha}+10^{2 \alpha}(6 n-18)+180^{\alpha}(12 n-24)+18^{2 \alpha}\left(9 n^{2}-33 n+30\right)$.

(5) $R R_{\alpha}(H D N 3[n])=\frac{\left(18 n^{2}-36 n+18\right)}{4^{2 \alpha}}+\frac{24}{28^{\alpha}}+\frac{(36 n-72)}{40^{\alpha}}+\frac{\left(36 n^{2}-108 n+84\right)}{72^{\alpha}}+\frac{12}{70^{\alpha}}+\frac{6}{126^{\alpha}}+$ $\frac{(6 n-18)}{10^{2 \alpha}}+\frac{(12 n-24)}{180^{\alpha}}+\frac{\left(9 n^{2}-33 n+30\right)}{18^{2 \alpha}}$.

(6) $\operatorname{SDD}(\operatorname{HDN} 3[n])=224 n^{2}-\frac{1510}{3} n+\frac{30487}{105}$.

(7) $H(H D N 3[n])=2 \times\left[\frac{1}{8}\left(18 n^{2}-36 n+18\right)+\frac{24}{11}+\frac{1}{14}(36 n-72)+\frac{1}{22}\left(36 n^{2}-108 n+84\right)+\frac{12}{17}+\frac{6}{25}+\right.$ $\left.\frac{1}{20}(6 n-18)+\frac{1}{28}(12 n-24)+\frac{1}{36}\left(9 n^{2}-33 n+30\right)\right]=\frac{91}{11} n^{2}-\frac{4637}{330} n+\frac{15959}{2550}$.

(8) $\operatorname{ISI}(H D N 3[n])=2\left(18 n^{2}-36 n+18\right)+\frac{672}{11}+\frac{40}{14}(36 n-72)+\frac{72}{22}\left(36 n^{2}-108 n+84\right)+\frac{840}{17}+\frac{756}{25}+$ $5(6 n-18)+\frac{180}{28}(12 n-24)+\frac{324}{36}\left(9 n^{2}-33 n+30\right)=\frac{2583}{11} n^{2}-\frac{5637}{11} n+\frac{115452}{425}$.

(9) $A Z(H D N 3[n])=\frac{4^{6}}{6^{3}}\left(18 n^{2}-36 n+18\right)+\frac{24 \times 28^{3}}{9^{3}}+\frac{40^{3}}{12^{3}}(36 n-72)+\frac{72^{3}}{20^{3}}\left(36 n^{2}-108 n+84\right)+\frac{12 \times 70^{3}}{15^{3}}+$ $\frac{6 \times 126^{3}}{23^{3}}+\frac{10^{6}}{18^{3}}(6 n-18)+\frac{180^{3}}{26^{3}}(12 n-24)+\frac{18^{6}}{34^{3}}\left(9 n^{2}-33 n+30\right)$.

Proof. One can directly derive the above topological indices by applying the respective derivation formulas over the proposed M-polynomial $M(H D N 3[n] ; x, y)$ as directed in the fifth column of Table 2 . These calculations are trivial and hence they are left to the ambitious reader.

Remark 5. In the paper [38], the general Randic index is calculated for the HDN3[n] for different values of $\alpha$. Instead of calculating this index individually, we can simply put these values of $\alpha$ in the above formula of $R_{\alpha}(H D N 3[n])$ to generate the respective values of the general Randic index.

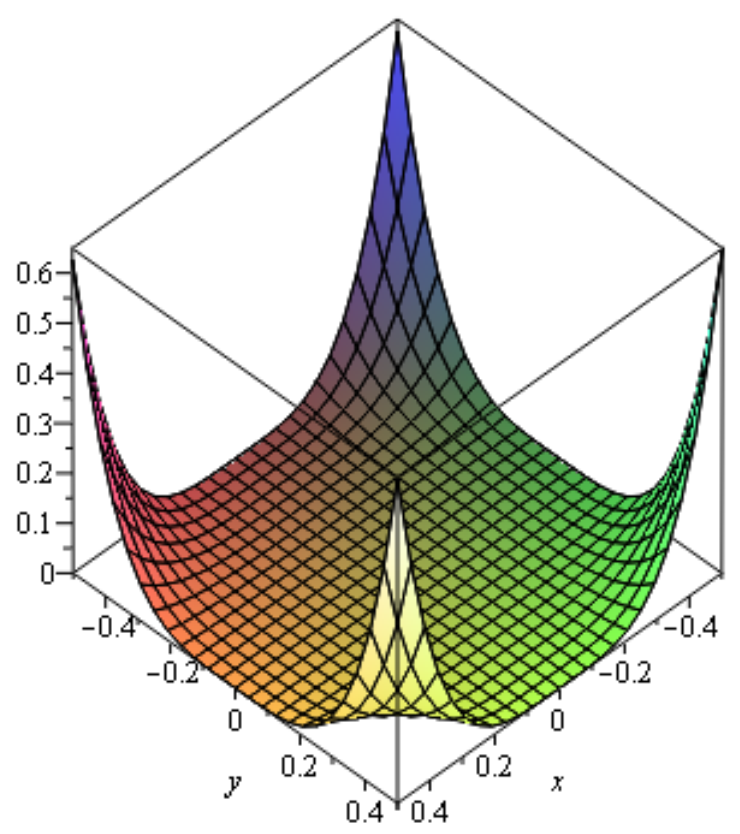

FIG. 2. The plot of the M-polynomial of $H D N 3[4]$ in region $-0.5 \leq x, y \leq 0.5$ 


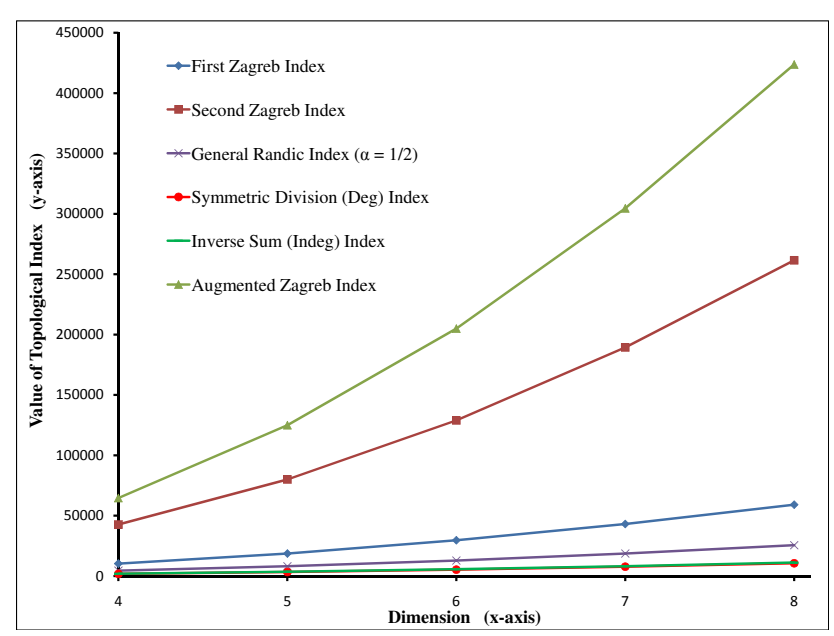

FIG. 3. A plot of first Zagreb, second Zagreb, general Randić $(\alpha=1 / 2)$, symmetric division (deg), inverse sum (indeg) and augmented Zagreb indices of $\operatorname{HDN} 3[n]$ for different values of $n(4 \leq n \leq 8)$

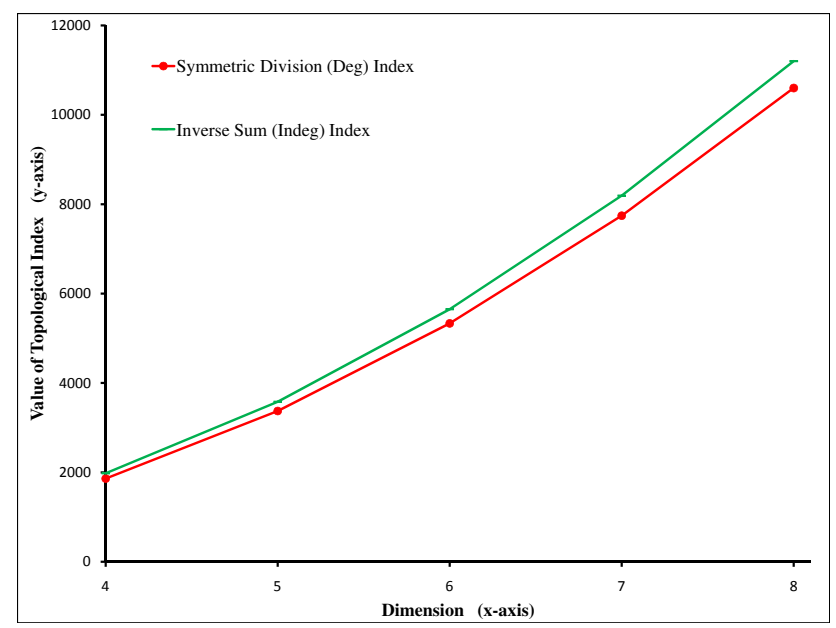

FIG. 4. A plot of only symmetric division (deg) and inverse sum (indeg) indices of $H D N 3[n]$ for different values of $n(4 \leq n \leq 8)$

\section{Experimental results related to the M-polynomial}

Fig. 2 pictorially models the M-polynomial $M(H D N 3[4] ; x, y)$ of the hex-derived network of type 3 of dimension $4(H D N 3[4])$ in the domain $-0.5 \leq x, y \leq 0.5$, by using Maple ${ }^{\mathrm{TM}} 13$ software.

Additionally, by observing the broad range of values of the degree-based topological indices of $H D N 3[n]$ for different dimensional values of $n(4 \leq n \leq 8)$, we plot the values of the first Zagreb, second Zagreb, general Randić ( $\alpha=1 / 2$ ), symmetric division (deg), inverse sum (indeg) and augmented Zagreb indices in Fig. 3, and the values of the modified second Zagreb, inverse Randić $(\alpha=1 / 2)$ and Harmonic indices in Fig. 5. The curves plotted in the Fig. 3 for the symmetric division (deg) and inverse sum (indeg) indices are almost overlapping and hence separately redrawn in Fig. 4. It is evident from the figures that the values of each of the topological indices are increasing when the dimensions are in increasing order. 


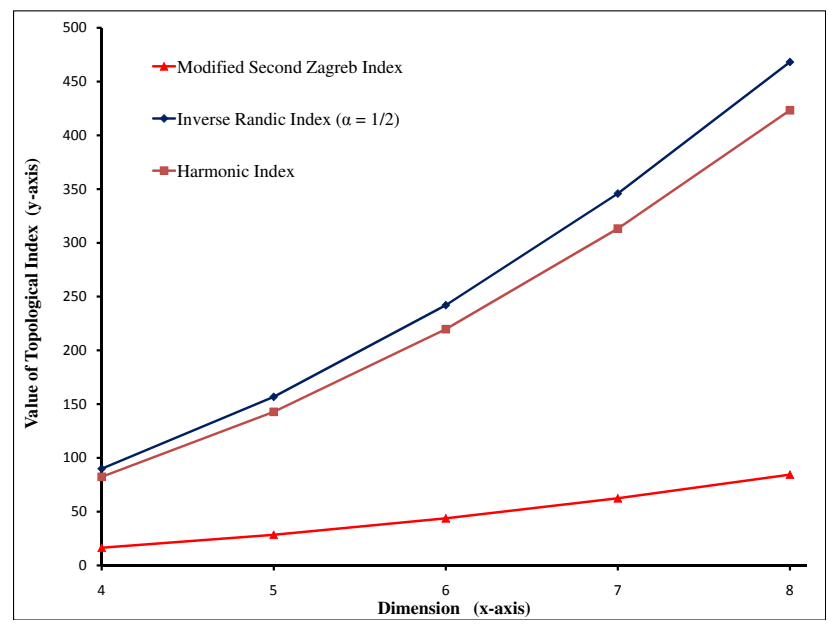

FIG. 5. A plot of modified second Zagreb, inverse Randić $(\alpha=1 / 2)$ and Harmonic indices of $H D N 3[n]$ for different values of $n(4 \leq n \leq 8)$

\section{Conclusion}

In this paper, we have considered the third type of hex-derived network of dimension $n(H D N 3[n])$ which has several applications in pharmaceutical sciences, electronics and communication networks. Instead of calculating the various degree-based topological indices separately, we have determined a general and closed-form of M-polynomial and thus directly derived the related degree-based topological indices of $H D N 3[n]$. Also, we have graphically represented the M-polynomial, and all the associated topological indices for different dimensions.

\section{References}

[1] West D. B. Introduction to Graph Theory. 2nd Edition, Prentice Hall., 2000.

[2] Hammack R., Imrich W., Klavžar S. Handbook of Product Graphs. 2nd Edition, CRC Press, Inc., Boca Raton, FL, USA, 2011.

[3] Estrada E. Randić index, irregularity and complex biomolecular networks. Acta Chimica Slovenica, 2010, 57, P. 597-603.

[4] García-Domenech R., Gálvez J., de Julián-Ortiz J. V., Pogliani L. Some new trends in chemical graph theory. Chemical Reviews, 2008, 108(3), P. $1127-1169$.

[5] Balaban A. T. Chemical applications of graph theory. Mathematical Chemistry. Academic Press., 1976.

[6] Trinajstić N. Chemical Graph Theory. Mathematical Chemistry Series. 2nd edition, CRC Press., 1992.

[7] Gross J. L., Yellen J., Zhang P. Handbook of Graph Theory. Discrete Mathematics and Its Applications. 2nd edition, Chapman and Hall/CRC., 2013.

[8] Gutman I. Degree-based topological indices. Croatica Chemica Acta, 2013, 86(4), P. 351-361.

[9] Balaban A. T. Highly discriminating distance-based topological index. Chemical Physics Letters, 1982, 89(5), P. 399-404.

[10] Pattabiraman K. Degree and distance based topological indices of graphs. Electronic Notes in Discrete Mathematics, 2017, 63, P. $145-159$.

[11] Khadikar P. V., Deshpande N. V., Kale P. P., Dobrynin A., Gutman I., Domotor G. The szeged index and an analogy with the wiener index. Journal of Chemical Information and Computer Sciences, 1995, 35(3), P. 547-550.

[12] Gutman I. The acyclic polynomial of a graph. Publications de I'Institut Mathématique, 1977, 22(36) (42), P. 63-69.

[13] Farrell E. J. An introduction to matching polynomials. Journal of Combinatorial Theory, Series B, 1979, 27(1), P. 75-86.

[14] Zhang H., Zhang F. The clar covering polynomial of hexagonal systems I. Discrete Applied Mathematics, 1996, 69(1-2), P. $147-167$.

[15] Gutman I. Some relations between distance-based polynomials of trees. Bulletin (Académie serbe des sciences et des arts. Classe des sciences mathématiques et naturelles. Sciences mathématiques), 2005, 30, P. 1-7.

[16] Kauffman L. H. A tutte polynomial for signed graphs. Discrete Applied Mathematics, 1989, 25(1-2), P. $105-127$.

[17] Hosoya H. On some counting polynomials in chemistry. Discrete Applied Mathematics, 1988, 19(1-3), P. $239-257$.

[18] Wiener H. Structural determination of paraffin boiling points. Journal of the American Chemical Society, 1947, 69(1), P. 17-20.

[19] Deutsch E., Klavžar S. M-polynomial and degree-based topological indices. Iranian Journal of Mathematical Chemistry, 2015, 6(2), P. 93102.

[20] Munir M., Nazeer W., Rafique S., Kang S. M. M-polynomial and degree-based topological indices of polyhex nanotubes. Symmetry, 2016, 8(12), P. 149.

[21] Munir M., Nazeer W., Rafique S., Kang S. M. M-polynomial and related topological indices of nanostar dendrimers. Symmetry, 2016, 8(9), P. 97.

[22] Munir M., Nazeer W., Nizami A. R., Rafique S., Kang S. M. M-polynomial and topological indices of titania nanotubes. Symmetry, 2016, 8(1-9), P. 117.

[23] Kwun Y. C., Munir M., Nazeer W., Rafique S., Kang S. M. M-polynomials and topological indices of V-phenylenic nanotubes and nanotori. Scientific Reports, P. 2017, 7(1), P. 1-9. 
[24] Kang S. M., Nazeer W., Zahid M. A., Nizami A. R., Aslam A., Munir M. M-polynomials and topological indices of hex-derived networks. Open Physics, 2018, 16(1), P. 394-403.

[25] Deng H., Yang J., Xia F. A general modeling of some vertex-degree based topological indices in benzenoid systems and phenylenes. Computers \& Mathematics with Applications, 2011, 61(10), P. 3017-3023.

[26] Gutman I., Trinajstić N. Graph theory and molecular orbitals. Total $\pi$-electron energy of alternant hydrocarbons. Chemical Physics Letters, 1972, 17(4), P. 535-538.

[27] Miličević A., Nikolić S., Trinajstić N. On reformulated zagreb indices. Molecular Diversity, 2004, 8, P. $393-399$.

[28] Bollobás B., Erdős P. Graphs of extremal weights. Ars Combinatoria, 1998, 50, P. 225-233.

[29] Amić D., Bešlo D., Lučić B., Nikolić S., Trinajstić N. The vertex-connectivity index revisited. Journal of Chemical Information and Computer Sciences, 1998, 38(5), P. 819-822.

[30] Vukičević D., Gašperov M. Bond additive modeling 1. adriatic indices. Croatica Chemica Acta, 2010, 83(3), P. $243-260$.

[31] Favaron O., Mahéo M., Saclé J. -F. Some eigenvalue properties in graphs (conjectures of graffiti-II). Discrete Mathematics, 1993, 111(1-3), P. 197-220.

[32] Furtula B., Graovac A., Vukičević D. Augmented zagreb index. Journal of Mathematical Chemistry, 2010, 48(2), P. 370-380.

[33] Randić M. Characterization of molecular branching. Journal of the American Chemical Society, 1975, 97(23), P. 6609-6615.

[34] Sedlar J., Stevanović D., Vasilyev A. On the inverse sum indeg index. Discrete Applied Mathematics, 2015, 184, P. $202-212$.

[35] Nocetti F. G., Stojmenovic I., Zhang J. Addressing and routing in hexagonal networks with applications for tracking mobile users and connection rerouting in cellular networks. IEEE Transactions on Parallel and Distributed Systems, 2002, 13(9), P. 963-971.

[36] Manuel P., Bharati R., Rajasingh I., Monica M C. On minimum metric dimension of honeycomb networks. Journal of Discrete Algorithms, 2008, 6(1), P. 20-27.

[37] Raj F. S., George A. On the metric dimension of HDN 3 and PHDN 3. 2017 IEEE International Conference on Power, Control, Signals and Instrumentation Engineering (ICPCSI), 2017, P. 1333-1336.

[38] Wei C. -C., Ali H., Binyamin M. A., Naeem M. N., Liu J.-B. Computing degree based topological properties of third type of hex-derived networks. Mathematics, 2019, 7(4), P. 368. 